\author{
Associate Professor Shouheng Tuo, PhD \\ School of Computer Science \& Technology, Xi'an University of \\ Posts \& Telecommunications, China \\ E-mail: tuo_sh@126.com \\ Associate Professor Hong He \\ College of Economics and Management, Xi'an University of \\ Posts \& Telecommunications, China \\ E-mail: hehongtbw@126.com
}

\title{
SOLVING COMPLEX CARDINALITY CONSTRAINED MEAN- VARIANCE PORTFOLIO OPTIMIZATION PROBLEMS USING HYBRID HS AND TLBO ALGORITHM
}

\begin{abstract}
Aiming to obtain maximum investment returns with minimum risk, portfolio selection with diversity is very necessary. However, the portfolio optimization selection is complex NP-complete problem. To address the portfolio optimization problem, a hybrid swarm intelligent optimization approach that combines harmony search (HS) and teaching-learning-based optimization (TLBO) is presented in this work. We introduce a Cardinality Constrained Mean-Variance $(C C M V)$ model which incorporates the boundary constraints (such as the bound of portfolio selection proportion of each asset and the number of assets) and considers transaction costs. To enhance the global optimization performance, an improved $H S$ and modified TLBO are synergistically performed using a dynamic selection strategy for balancing the global exploration power and the local exploitation power. The experimental results on five data sets (HangSeng, DAX 100, FTSE 100, $S \& P 100$, and Nikkei 225) demonstrate that the proposed algorithm is effective and efficient in solving complex portfolio selection problems.

Keywords: Portfolio optimization problems, Cardinality Constrained MeanVariance, Harmony search, Teaching-Learning-Based Optimization.
\end{abstract}

\section{JEL Classification: G11}

\section{Introduction}

In recent years, the global economy runs unsteadily owing to the influence of financial storm onset in 2008. As rising of prices, devaluing of currency, and very low profit in traditional bank storage, organizations and people have begun to seek new investments for capital appreciation. In order to obtain high return of investment and reduce simultaneously the investment risk, diversifying economic investments, such as common stocks, domestic, foreign bond indices, foreign cash, real estate, commodities and so on, must be considered by the investors. As a consequence, it is of importance for optimizing the selection of portfolio from large amount of

DOI: $10.24818 / 18423264 / 52.3 .18 .16$ 
Shouheng Tuo, Hong He

investment products in which some of them has high expected return but high risk, others has low returns with low risk. On this issue, there are two problems to be resolved: first is to establish a feasible portfolio selection model, second is to obtain the optimal portfolio selection by solving the portfolio model. For the first problem, researchers have put forward many portfolio optimization theory models. Markowitz [1] presented the first mathematical model, named Mean-Variance (MV) model, of the portfolio optimization problems. Then the MV model is improved to take more realistic features into consideration (see [2-5]), the MV model assumes that the returns on all assets obey the normal distribution and the investors expect to obtain maximum returns with minimum risk, which does not take transaction cost into consideration. Recently cardinality constraints mean-variance (CCMV) model (see [6-8]) gets attention more and more, which includes the weight constraints of each asset and the number of assets selected in a portfolio.

In reality, most of the available portfolio selection models are large scale combination optimization problems, which have enormous computational burden. The Markowitz MV portfolio model can be formulated as quadratic programming problem which can be solved using quadratic optimization techniques (such as quadratic programming). However, the quadratic programming cannot be employed to solve the CCMV model owing to including the cardinality constraints, which leads to the MV model be a no-convex problem. The CCMV model is NP-complete problem whose computation cost has an exponential increase as the increase of the number of available assets. Although some improved models aim to decrease the computational burden of the Markowitz model, such as mean absolute deviation model [9-10], meanwhile the models are simplified, which deviates from the realistic application.

In this work, the goal is to solve complex CCMV models without simplification using an intelligent hybrid optimization algorithm, named HS-TLBO, which combines harmony search (HS) [11] and teaching-learning-based Optimization (TLBO) for improving the global search performance. The HS and TLBO are complementary each other, where HS has very strong global exploration power; TLBO is very outstanding for finding high-precision globally optimal solution when the population has gathered into global optimal region.

\section{CCMV portfolio optimization model}

The CCMV model has two objectives: maximum the investment return and minimum the investment risk, as follows.

$$
\begin{aligned}
& f_{1}(\mathbf{x})=\max _{\mathbf{x}} \mathbf{x}^{\mathrm{T}} \boldsymbol{\mu}-\phi(\mathbf{x})=\max _{\boldsymbol{x}_{i}} \sum_{i=1}^{n}\left(x_{i} u_{i}-\phi_{i}\left(x_{i}\right)\right) \\
& f_{2}(\mathbf{x})=\min _{\mathbf{x}} \mathbf{x}^{\mathrm{T}} \sum \mathbf{x}=\min \sum_{i=1}^{n} \sum_{j=1}^{n} x_{i} x_{j} \sigma_{i j}
\end{aligned}
$$


Solving Complex Cardinality Constrained Mean-variance Portfolio Optimization Problems Using Hybrid HS and TLBO Algorithm

Subject to

$$
\begin{aligned}
& \sum_{i=1}^{n} z_{i}=K,\left(z_{i}=\left\{\begin{array}{ll}
1 & \text { if } x_{i} \neq 0 \\
0 & \text { otherwise }
\end{array}\right)\right. \\
& \sum_{i=1}^{n} x_{i}=1,\left(x_{i}^{L} z_{i} \leq x_{i} \leq x_{i}^{U} z_{i}\right)
\end{aligned}
$$

Where $f_{1}(\mathbf{x})$ and $f_{2}(\mathbf{x})$ are the objective functions of expected return and investment risk, respectively. $n$ is the number of total assets available, $\mathbf{x}=\left(x_{1}, x_{2}, \cdots, x_{n}\right)$ denotes the vector of portfolio weights that are the percentages of wealth invested in every asset. $u_{i}(i=1,2, \cdots, n)$ represents the expected return rate of $i^{\text {th }}$ asset. $\sum$ is a $n \times n$ positive semi-definite symmetric matrix in which $\sigma_{i j}(i=1,2, \cdots, n ; j=1,2, \cdots, n)$ denotes the covariance between the $\mathrm{i}^{\text {th }}$ and $\mathrm{j}^{\text {th }}$ asset returns. $\phi_{i}\left(x_{i}\right)(i=1,2, \cdots, n)$ is the transaction costs incurred on buying or selling in the $\mathrm{i}^{\text {th }}$ security, and $\phi(\mathbf{x})$ denotes the total transaction costs on the portfolio. $K$ is the desired number of assets in the portfolio. $x_{i}^{L}\left(0 \leq x_{i}^{L} \leq 1\right)$ and $x_{i}^{U}\left(0 \leq x_{i}^{U} \leq 1\right)$ separately denote the lower bound and upper bound of $x_{i}$, which are the lowest limit and the maximum limit on the proportion of the $\mathrm{i}^{\text {th }}$ asset.

\section{Related work}

CCMV portfolio selection model is a complex two-objective optimization problem, which cannot be resolved using traditional mathematical optimization methods owing to the cardinality constraints and characteristic of non-convex. Many methods have been presented to solve the CCMV model. However, the performance of many of them is not satisfactory in solving large scale portfolio selection problems. In recent years, heuristic search algorithms and swarm intelligent optimization algorithms are applied to address this problem. Bertsimas, D et al. employed branch-and-bound technique to decrease the computation burden [13]. Bienstock, D used the branch-and-cut method to solve the CCMV model [14]. In literature [15], Genetic algorithm (GA), Simulated annealing (SA) and Tabu search (TS) are respectively employed into the solving of CCMV portfolio selection problems. Soleimani, H. et al adopted GA to address Markowitz-based portfolio selection with minimum transaction lost, cardinality constraints. In [17-18], Particle swarm optimization (PSO) and improved PSO are used to find optimal portfolio selection. Although these methods have demonstrated the effectiveness for solving CCMV model, they still have some disadvantages for solving complex CCMV models, such as high computation cost, slow speed, trapping into local search easily and so on. To tackle these drawbacks, we employ two new intelligent optimization

DOI: $10.24818 / 18423264 / 52.3 .18 .16$ 
Shouheng Tuo, Hong He

algorithms: harmony search (HS) and teaching-learning-based optimization (TLBO), which have strong robustness for solving high-dimensional optimization problems.

\section{A Hybrid Optimization Approach To CCMV Portfolio Selection}

\subsection{Hybrid HS-TLBO algorithm}

The pseudo code of HS-TLBO algorithm is shown in Fig.1.

(1) Initializing parameters:

Population size $\mathbf{N P}=\mathbf{1 0}$.

Maximum function evaluation times $\mathbf{T}_{\max }=2000 * \mathbf{N P}$.

HMCR $=0.98$ (HMCR is the harmony memory consideration rate of HS).

$\mathbf{P A R}=0.35$ (PAR is pitch adjustment rate of HS).

Current iteration $\mathbf{t}=\mathbf{1}$; selection probability $\mathbf{S P}=\mathbf{0 . 9}$ of algorithms (HS and TLBO).

$\mathrm{sc} 1=0, \mathrm{sc} 2=0, \mathbf{c}=0, \mathbf{C}=1000$;

(2) Initializing the population in feasible space randomly, as follow

$X(i, j)=x_{j}^{L}+r_{j} \times\left(x_{j}^{U}-x_{j}^{L}\right), i=1,2, \ldots, n ; j=1,2, \ldots, n$

Where $x_{j}^{L}$ and $x_{j}^{U}$ are the lowest limit and the maximum limit on the proportion of the $\mathrm{j}^{\text {th }}$ asset.

(3) If $\operatorname{rand}(0,1)<S P$, then else

perform modified HS(see Fig.1), $\mathrm{sc} 1=\mathrm{sc} 1+1$;

executemodified TLBO (see Fig.2)algorithm, sc2=sc2+1.

(4) $\mathbf{c}=\mathbf{c}+\mathrm{NP}$

If $\mathbf{c}<\mathbf{C}$, then $\mathbf{t}=\mathbf{t}+\mathrm{NP}$.

else

$\mathbf{c}=0, \mathrm{~S} 1=\mathrm{c} 1 /(\mathbf{S P} * \mathbf{C}), \mathrm{S} 2=\mathrm{c} 2 /((1-\mathbf{S P}) * \mathbf{C})$.

$\mathbf{S P}=\mathrm{S} 1 /(\mathrm{S} 1+\mathrm{S} 2)$.

If $\mathrm{t}<\mathrm{T}_{\max } / 2$, then $\mathrm{SP}=\min (\max (0.9, \mathrm{SP}), 0.98)$,

else

$\mathrm{SP}=\min (\max (0.3, \mathrm{SP}), 0.99)$.

$\mathbf{t}=\mathbf{t}+\mathbf{N P}$.

(5) If $\mathbf{t}<\mathbf{T}_{\max }$, go to $\operatorname{step}(3)$

elseend the algorithm.

Figure1. The pseudo code of HS-TLBO

Harmony search (HS) is proposed in 2001 by Z.W. Geen et al, it mimics the improvisation process of jazz musicians [11]. HS has robustly global exploration 
Solving Complex Cardinality Constrained Mean-variance Portfolio Optimization Problems Using Hybrid HS and TLBO Algorithm

power in solving complex multi-modal optimization problems but low precision of optimal solution. Teaching-learning-based optimization (TLBO) [12] imitates the learning process of a learner in our life, which consists of two stages: teaching phase and learning phase, in which each learner enhances ability by learning from teacher in teaching phase and learning from other learners in learning stage. The TLBO has very fast speed for solving high-dimensional optimization problems. In this work, we aim to integrate the advantages of the two methods together for solving complex CCMV portfolio selection problems.

To integrate the HS and the TLBO well, we modify the HS and TLBO algorithms, respectively.

\subsection{Modified HS algorithm}

In the modified HS algorithm, the parameters PAR and $f w$ are dynamically changed with the increase of iteration. The iteration process of modified HS is displayed in Fig.2.

In Fig.2, rand $(0,1)$ generates a random number of uniform distributions between 0 and 1 . The idxworst is the index of worst harmony in harmony memory. $R$ denotes a random integer in range $[1, \mathrm{NP}]$. In order to balance the exploration power and the exploitation power, the parameter $P A R$ (see Equation (3)) and $f w$ (see Equation (4) and (5)) in the modified HS are dynamically changed with the increasing of iteration, as follows.

$$
\begin{aligned}
& P A R=P A R_{\min }+\left(P A R_{\max }-P A R_{\min }\right) \times \frac{t}{T_{\max }} \\
& f w(i)=\left\{\begin{array}{l}
f w_{\max }(i) \times \exp \left(\left(\frac{t}{T_{\max }}\right)^{2} \times \log \frac{f w_{\min }(i)}{f w_{\max }(i)}\right), t<T_{\max }, i=1,2, \ldots, D \\
\left|x_{i}^{R_{a}}-x_{i}^{\text {new }}\right|, t \geq T_{\max }
\end{array}\right. \\
& f w 2(i)=\left\{\begin{array}{l}
\left(x_{i}^{U}-x_{i}^{L}\right) / 1000, f w(i)<10^{-15} \| f w(i)>\left(x_{i}^{U}-x_{i}^{L}\right) / 1000 \\
\left|x_{i}^{R_{a}}-x_{i}^{\text {new }}\right|, \text { otherwise }
\end{array}\right.
\end{aligned}
$$

DOI: 10.24818/18423264/52.3.18.16 


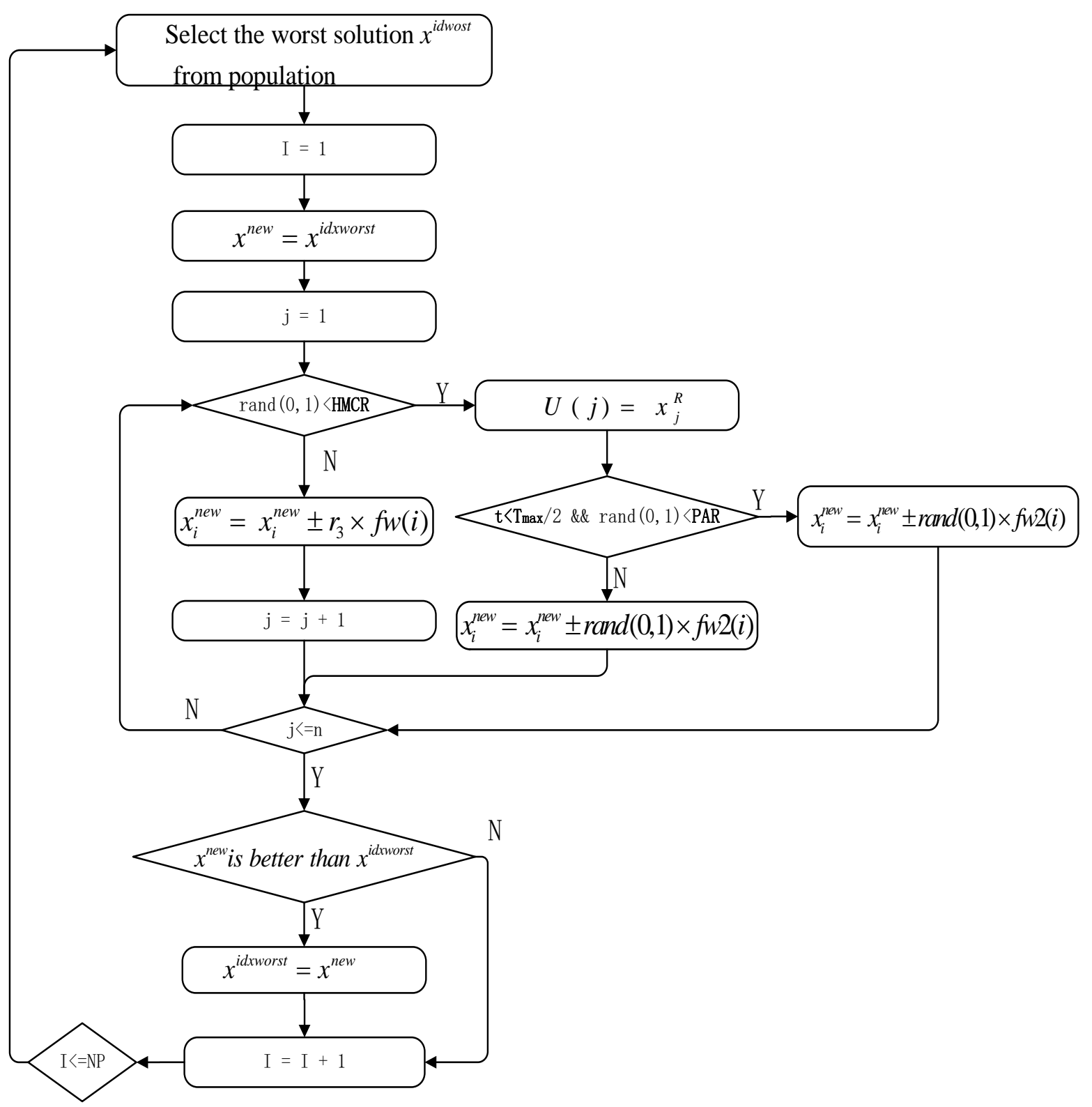

Figure 2. Flow chart of modified HS algorithm 
Solving Complex Cardinality Constrained Mean-variance Portfolio Optimization Problems Using Hybrid HS and TLBO Algorithm

\subsection{Modified TLBO algorithm}

Unlike the standard TLBO, in the modified TLBO algorithm, each learner only selects part of subjects to learn from teacher or other learners in each iteration. The iteration process of modified TLBO is shown inFig.3.

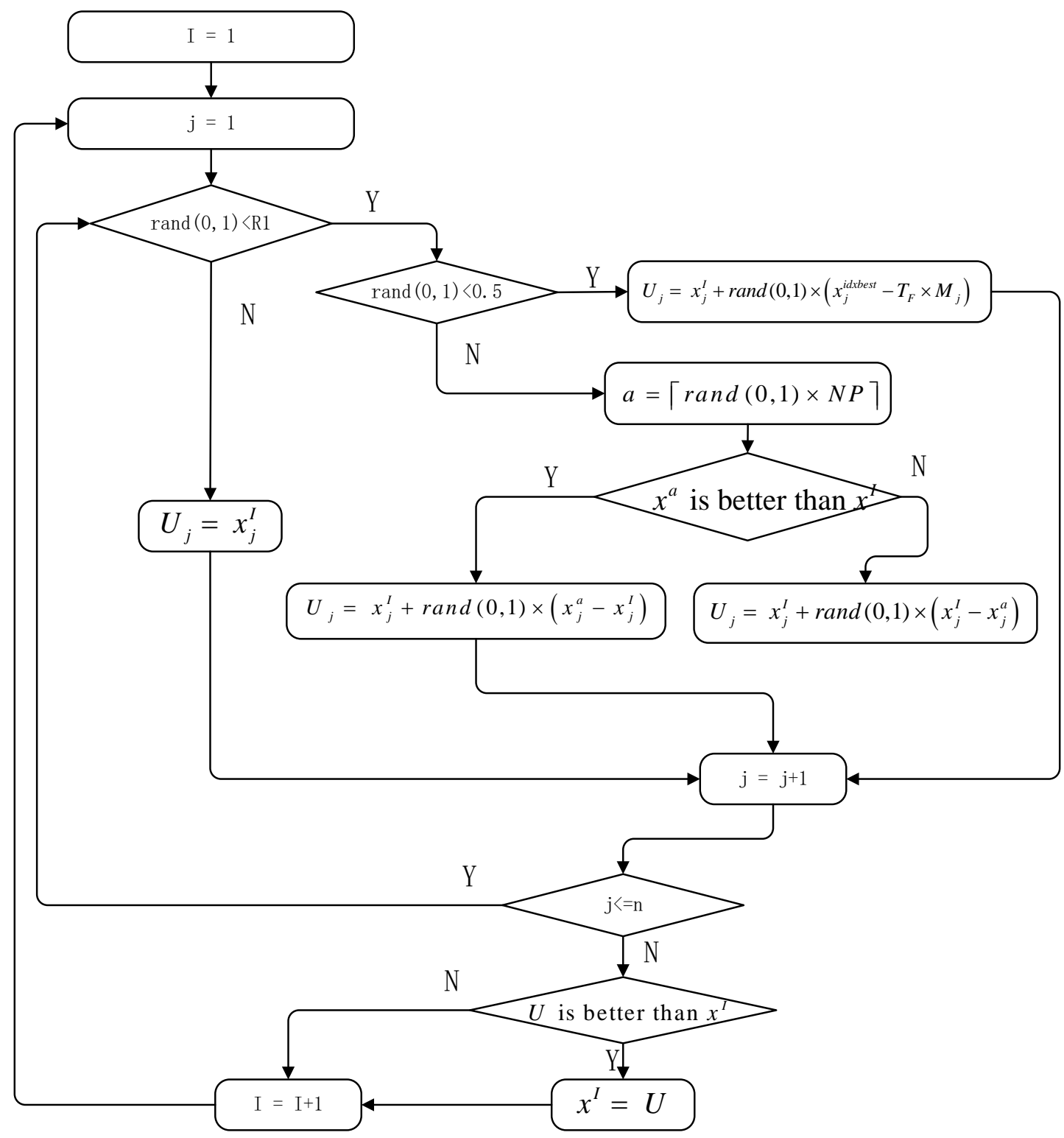

Figure 3. The flow chart of modified TLBO

DOI: $10.24818 / 18423264 / 52.3 .18 .16$ 


$$
R 1=\left\{\begin{array}{c}
1, \quad n \leq 30 \\
\min \left(0.6, \frac{30}{n}\right)\left(1+\left(1-\frac{\mathbf{t}}{\mathbf{T}_{\mathbf{m a x}}}\right)^{2}\right), \text { otherwise }
\end{array}\right.
$$

In Fig.3, $R 1$ (see equation 6) is the selection probability for learners selecting some subjects to learn knowledge from teacher or other learners. The $R 1$ is equal to 1 when $n<=30$, which means that the learner learns from others on all $n$ subjects; however, when the number of total assets is larger than 30 , the $R 1$ will decrease correspondingly, which aims to increase the success rate that new generated solution is superior to old solution. $M_{j}=x_{j}^{r}(j=1,2, \cdots, n)$, where $r$ is a random integer between 1 and NP. follows.

The primary difference between standard TLBO and modified TLBO is as

(1) In modified TLBO, it is a combination vector in which each subject $M(i)$ is randomly chosen from the $\mathrm{i}^{\text {th }}$ subject of all learners.

(2) In each iteration. TLBO performs teacher phase and learner phase, respectively. The modified TLBO only randomly chooses either teacher phase or learner phase to perform.

(3) In standard TLBO, all dimensions of $x^{\text {new }}$ are produced by learning from teacher or other one learner. Whereas, in modified TLBO, only some dimensions of $x^{\text {new }}$ are generated by learning from teacher or other one learner, and other dimensions is inherited from $x^{\text {old }}$ directly, the reason for this is that an excellent learner is also imperfect on some subjects. Therefore, selective learning on some subjects is more effective for improving knowledge level of learner than learning all subjects from one learner.

(4) As we known, in our real lives, selective learning from multiple excellent learners on some subjects is more effective for improving our knowledge level than learning all subjects only from one excellent learner. As a consequence, in the modified TLBO, the learner on each subject selects one other learner from class for learning new knowledge.

\section{Solving CCMV model using hybrid HS-TLBO}

There are two objectives (maximizing the portfolio return and minimizing the investment risk) to be considered for the CCMV model. Some algorithms [20- 
Solving Complex Cardinality Constrained Mean-variance Portfolio Optimization Problems Using Hybrid HS and TLBO Algorithm

21] use the multi-objective optimization technique to solve it. In this work, we employ the proposed HS-TLBO to solve the complex CCMV model, and weighted single objective CCMV model is used to find the optimal portfolio selection, which aims to find the efficient frontier of Pareto optimal selections in terms of weighted coefficient $\lambda \in[0,1]$. The single objective CCMV model is as equation (7).

$$
\max f(\mathrm{x})=\lambda f_{1}(\mathrm{x})-(1-\lambda) f_{2}(\mathrm{x})
$$

In equation (7), the value $\lambda=1$ corresponds to maximize the expected return of portfolio selections without the consideration of portfolio risk. The value $\lambda=0$ means to minimize the portfolio risk without regard to the expected portfolio return. The portfolio returns and risks of optimal solutions based on different value of $\lambda$ will construct an efficient frontier of optimal portfolio selections.

In this study, we consider the transaction cost in which the function $\phi_{i}\left(x_{i}\right)$ is defined as equation (8).

$$
\phi_{i}\left(x_{i}\right)=\left\{\begin{array}{cc}
0 & , x_{i}=0 \\
F_{i}+\alpha_{i} x_{i} & , x_{i}>0
\end{array}\right.
$$

Where $F_{i}(i=1,2, \ldots, \mathrm{n})$ is the fixed transaction cost that are incurred in any selected assets , $\alpha_{i} x_{i}$ denotes the variable transaction cost for $\mathrm{i}^{\text {th }}$ selected asset, $\alpha_{i}$ is the proportional transaction costs of the $\mathrm{i}^{\text {th }}$ asset.

In HS-TLBO, the solution $\mathrm{x}^{\mathrm{j}}=\left(x_{1}^{j}, x_{2}^{j}, \ldots, x_{n}^{j}\right)$ in population represents an investment selection; the objective function is expressed as equation (7). We employ the method of literatures [17, 22-23] to handle the constraints. The HS-TLBO flow chart for optimizing the portfolio selection is shown in Fig.4.

In Fig.4, $\mathrm{T}$ and Tmax represent the number of current iteration and the number of maximum iteration, respectively. step denotes the step of $\lambda$ that increases from 0 to 1 . For each $\lambda$, HS-TLBO will obtain an optimal portfolio selection that is recorded.

DOI: 10.24818/18423264/52.3.18.16 
Shouheng Tuo, Hong He

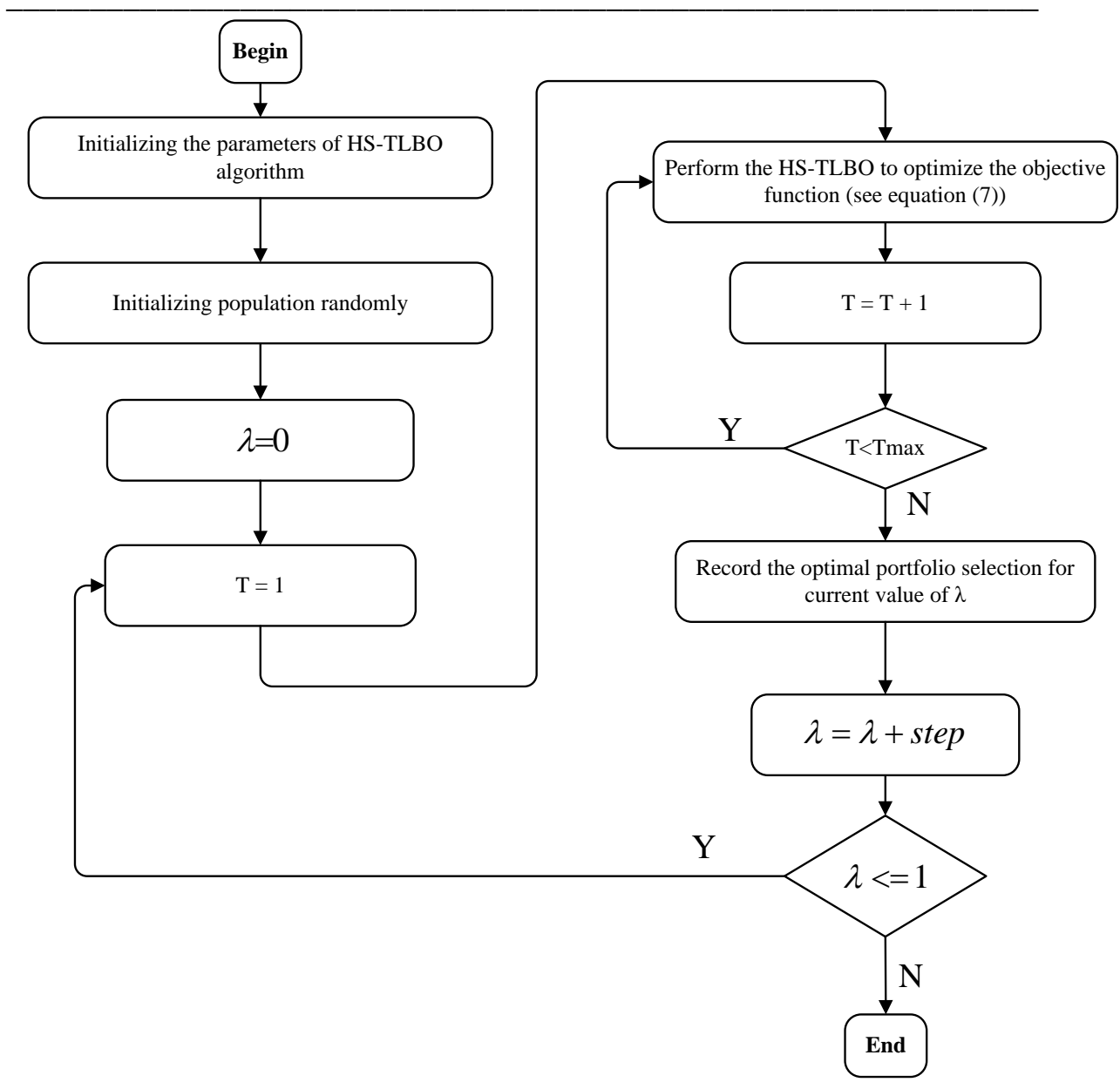

Figure 4. The HS-TLBO flow chart for optimizing the portfolio selection.

\section{Numerical experiments}

To investigate the performance of proposed HS-TLBO algorithm, we apply it to five real datasets (HangSeng, DAX100, FTSE100, S\&P100 and Nikkei) (see http://people.brunel.ac.uk/ mastjjb/jeb/orlib/portinfo.html ). The numerical experiments are performed on three conditions:

(1) Unconstraint. The portfolio proportion, desired number of investment assets are not constrained, and the transaction costs (TC) are not considered in this experiment. 
Solving Complex Cardinality Constrained Mean-variance Portfolio Optimization Problems Using Hybrid HS and TLBO Algorithm

(2) Constraint without considering the transaction costs (TC). The transaction costs are not considered in this case.

(2.1) Constraint1: The portfolio proportion of each asset $x_{i}^{L}=0.0$ and $x_{i}^{U}=1$; the desired number of portfolio selection assets $K=10$.

(2.2) Constraint2: The portfolio proportion of each asset $x_{i}^{L}=0.01$ and $x_{i}^{U}=1$; the desired number of portfolio selection assets $K=10$.

(3) Constraint considering the transaction costs. The fixed transaction cost are set as one thousandth of one millimeter of mean expected return and the variable transaction cost of $\mathrm{i}^{\text {th }}$ asset is equal to $0.003 x_{i} m_{i}$, where $m_{i}$ denotes the expected return of $i^{\text {th }}$ asset.

(3.1) Constraint1 considering TC: The bound of portfolio proportion of each asset $x_{i}^{L}=0.0$ and $x_{i}^{U}=1$; the desired number of portfolio selection assets $K=10$.

(3.2) Constraint 2 considering TC: The bound of portfolio proportion of each asset $x_{i}^{L}=0.0$ and $x_{i}^{U}=1$; the desired number of portfolio selection assets equals 10 .

In the experiments with three constraint conditions, the value of $\lambda$,expressed as $\lambda_{j}(=j \times$ step $, j=1,2, \cdots, 1 /$ step $)$, increases from 0 to 1 with step $=0.05$. We employ mean Euclidian distance $(M E D)$, variance of returns error $(V R E)$, and mean return error (MRE) as performance indexes that are defined as equations (9)-(11)[17, 22-23]. The experimental results of HS-TLBO are compared with those of four stateof-the-art intelligent algorithms (PSO, GA, SA, TS), which are summarized in Table 1-Table2.

$$
\begin{gathered}
M E D=\frac{1}{N} \sum_{j=1}^{N} \sqrt{\left(v_{i_{j}}^{s}-v_{j}^{h}\right)^{2}+\left(r_{i_{j}}^{s}-r_{j}^{h}\right)^{2}} \\
V R E=\frac{1}{N}\left(\sum_{j=1}^{N} \frac{100\left|v_{i_{j}}^{s}-v_{j}^{h}\right|}{v_{j}^{h}}\right) \\
M R E=\frac{1}{N}\left(\sum_{j=1}^{N} \frac{100\left|r_{i_{j}}^{s}-r_{j}^{h}\right|}{r_{j}^{h}}\right)
\end{gathered}
$$

DOI: 10.24818/18423264/52.3.18.16 
Shouheng Tuo, Hong He

where $r_{i}^{s}$ and $v_{i}^{s}(\mathrm{i}=1,2, \ldots, 2000)$ denote the variance (risk) and mean return in the standard efficient frontier without constraint conditions, $v_{j}^{h}$ and $r_{j}^{h}$ represent the minimum variance and maximum return of optimal portfolio selection obtained by search algorithm on $\lambda_{j}$, respectively. $N$ is the number of $\lambda$. In this work, $N=(1 /$ step) $+1 . i_{j}=\underset{i=1,2, \cdots, 2000}{\arg \min }\left(\sqrt{\left(v_{i}^{s}-v_{j}^{h}\right)^{2}+\left(r_{i}^{s}-r_{j}^{h}\right)^{2}}\right),(j=0,2, \cdots, N-1)$, which denotes the closest point in the standard efficient frontier to the Pareto optimal efficient frontier obtained by optimization algorithm.

In this work, we employ the same method of constraint handle as the literature [23] which can handle the boundary constraint of the portfolio proportion and the desired number of portfolio selection assets very well.

To illustrate that constraint of the number of portfolio selection assets bounded constraints and considering of transaction costs, we present the comparison of efficient frontiers for different constraint conditions in Fig.5 - Fig.9.

Table 1.The experimental results of 5 algorithms for unconstraint CCMV model

\begin{tabular}{|c|c|c|c|c|c|c|c|c|c|c|c|c|c|c|c|}
\hline \multirow{2}{*}{ Alg } & \multicolumn{3}{|c|}{ HangSeng (31) } & \multicolumn{3}{|c|}{ DAX100 (85) } & \multicolumn{3}{|c|}{ FTSE100 (89) } & \multicolumn{3}{|c|}{ S\&P100 (98) } & \multicolumn{3}{|c|}{ Nikkei(225) } \\
\hline & MED & VRE & MRE & MED & VRE & MRE & MED & VRE & MRE & MED & VRE & MRE & MED & VRE & MRE \\
\hline GA & 5.9E-04 & 2.9E-01 & $1.1 \mathrm{E}-01$ & $1.2 \mathrm{E}-03$ & $3.1 \mathrm{E}-01$ & $1.2 \mathrm{E}-01$ & $3.0 \mathrm{E}-04$ & $5.0 \mathrm{E}-01$ & $5.7 \mathrm{E}-02$ & $6.2 \mathrm{E}-04$ & $6.1 \mathrm{E}-01$ & 2.1E-01 & $1.5 \mathrm{E}-03$ & 2.1E-01 & $9.3 \mathrm{E}-01$ \\
\hline PSO & 7.4E-04 & $3.9 \mathrm{E}-01$ & $1.3 \mathrm{E}-01$ & $1.4 \mathrm{E}-03$ & $3.9 \mathrm{E}-01$ & $1.3 \mathrm{E}-01$ & 3.3E-04 & 5.4E-01 & $6.4 \mathrm{E}-02$ & $7.9 \mathrm{E}-04$ & 6.9E-01 & $2.5 \mathrm{E}-01$ & $2.9 \mathrm{E}-04$ & 4. $3 \mathrm{E}-01$ & 1.4E-01 \\
\hline TS & $6.0 \mathrm{E}-04$ & 2.9E-01 & $1.1 \mathrm{E}-01$ & $1.2 \mathrm{E}-03$ & $2.9 \mathrm{E}-01$ & $1.1 \mathrm{E}-01$ & 3.2E-04 & 7.0E-01 & $5.8 \mathrm{E}-02$ & $6.2 \mathrm{E}-04$ & $1.0 \mathrm{E}+00$ & $1.3 \mathrm{E}-01$ & $1.5 \mathrm{E}-04$ & 2.2E-01 & 7.4E-02 \\
\hline SA & $6.1 \mathrm{E}-04$ & $2.9 \mathrm{E}-01$ & $1.1 \mathrm{E}-01$ & $1.2 \mathrm{E}-03$ & $2.9 \mathrm{E}-01$ & $1.1 \mathrm{E}-01$ & 3.3E-04 & 6.7E-01 & $5.8 \mathrm{E}-02$ & $6.2 \mathrm{E}-04$ & $9.5 \mathrm{E}-01$ & $1.5 \mathrm{E}-01$ & $1.9 \mathrm{E}-04$ & 2.1E-01 & 7.2E-02 \\
\hline HS-TLBO & 7.8E-07 & $1.9 \mathrm{E}-02$ & 8.9E-03 & $1.8 \mathrm{E}-06$ & $9.6 \mathrm{E}-02$ & $1.0 \mathrm{E}-02$ & 4.8E-07 & $2.4 \mathrm{E}-02$ & $\underline{5.9 \mathrm{E}-03}$ & $1.6 \mathrm{E}-06$ & 7.3E-02 & $1.1 \mathrm{E}-02$ & 8.3E-07 & $6.4 \mathrm{E}-02$ & $1.3 \mathrm{E}-02$ \\
\hline
\end{tabular}

Table 2.The experimental results of 5 algorithms for constraint CCMV model

\begin{tabular}{|c|c|c|c|c|c|c|c|c|c|c|c|c|c|c|c|}
\hline \multirow{2}{*}{ Alg } & \multicolumn{3}{|c|}{ HangSeng (31) } & \multicolumn{3}{|c|}{ DAX100 (85) } & \multicolumn{3}{|c|}{ FTSE100 (89) } & \multicolumn{3}{|c|}{ S\&P100 (98) } & \multicolumn{3}{|c|}{ Nikkei(225) } \\
\hline & MED & VRE & MRE & MED & VRE & MRE & MED & VRE & MRE & MED & VRE & MRE & MED & VRE & MRE \\
\hline GA for constraint 2 & $3.9 \mathrm{E}-3$ & $1.7 \mathrm{E}+0$ & $6.1 \mathrm{E}-1$ & $7.6 \mathrm{E}-3$ & $1.8 \mathrm{E}+0$ & $6.6 \mathrm{E}-1$ & $2.0 \mathrm{E}-3$ & $2.9 \mathrm{E}+0$ & $3.3 \mathrm{E}-1$ & $4.1 \mathrm{E}-3$ & $3.5 \mathrm{E}+0$ & $1.2 \mathrm{E}+0$ & $9.9 \mathrm{E}-3$ & $1.2 \mathrm{E}+0$ & $5.3 \mathrm{E}+0$ \\
\hline $\begin{array}{l}\text { PSO for } \\
\text { constraint2 }\end{array}$ & $4.9 \mathrm{E}-3$ & $2.2 \mathrm{E}+0$ & $7.4 \mathrm{E}-1$ & $9.0 \mathrm{E}-3$ & $2.2 \mathrm{E}+0$ & $7.4 \mathrm{E}-1$ & $2.2 \mathrm{E}-3$ & $3.1 \mathrm{E}+0$ & $3.6 \mathrm{E}-1$ & $5.2 \mathrm{E}-3$ & $3.9 \mathrm{E}+0$ & $1.4 \mathrm{E}+0$ & $1.9 \mathrm{E}-3$ & $2.4 \mathrm{E}+0$ & 8.0E-1 \\
\hline TS for constraint 2 & $4.0 \mathrm{E}-3$ & $1.7 \mathrm{E}+0$ & $6.1 \mathrm{E}-1$ & $8.2 \mathrm{E}-3$ & $1.7 \mathrm{E}+0$ & $6.1 \mathrm{E}-1$ & $2.1 \mathrm{E}-3$ & $4.0 \mathrm{E}+0$ & $3.3 \mathrm{E}-1$ & $4.1 \mathrm{E}-3$ & $5.7 \mathrm{E}+0$ & 7.1E-1 & $1.0 \mathrm{E}-3$ & $1.2 \mathrm{E}+0$ & 4.2E-1 \\
\hline SA for constraint 2 & $4.0 \mathrm{E}-3$ & $1.7 \mathrm{E}+0$ & $6.2 \mathrm{E}-1$ & $7.8 \mathrm{E}-3$ & $1.7 \mathrm{E}+0$ & $6.2 \mathrm{E}-1$ & $2.2 \mathrm{E}-3$ & $3.8 \mathrm{E}+0$ & $3.3 \mathrm{E}-1$ & 1.1E-3 & $5.4 \mathrm{E}+0$ & $8.4 \mathrm{E}-1$ & $1.2 \mathrm{E}-3$ & $1.2 \mathrm{E}+0$ & 4.1E-1 \\
\hline $\begin{array}{l}\text { HS-TLBO for } \\
\text { constraint } 1\end{array}$ & 7.9E-5 & $\underline{1.7 \mathrm{E}+0}$ & $6.1 \mathrm{E}-1$ & $1.5 \mathrm{E}-4$ & $\underline{1.3 \mathrm{E}+0}$ & $1.3 \mathrm{E}+0$ & $3.9 \mathrm{E}-5$ & $3.1 \mathrm{E}+0$ & $3.2 \mathrm{E}-1$ & $\underline{1.0 \mathrm{E}-4}$ & $\underline{8.2 \mathrm{E}+0}$ & $\underline{7.8 \mathrm{E}-1}$ & $\underline{6.6 \mathrm{E}-5}$ & $5.2 \mathrm{E}+0$ & $1.4 \mathrm{E}+0$ \\
\hline $\begin{array}{l}\text { HS-TLBO for } \\
\text { constraint2 }\end{array}$ & $\underline{6.6 \mathrm{E}-5}$ & $4.2 \mathrm{E}+0$ & $7.5 \mathrm{E}-1$ & $6.2 \mathrm{E}-6$ & $2.0 \mathrm{E}+0$ & $\underline{1.3 \mathrm{E}-2}$ & $\underline{9.3 \mathrm{E}-6}$ & $\underline{2.5 \mathrm{E}+0}$ & $\underline{2.4 \mathrm{E}-2}$ & $2.3 \mathrm{E}-4$ & $1.3 \mathrm{E}+1$ & $2.0 \mathrm{E}+0$ & $6.7 \mathrm{E}-5$ & $4.5 \mathrm{E}+0$ & $1.4 \mathrm{E}+0$ \\
\hline $\begin{array}{l}\text { HS-TLBO for } \\
\text { constraint } 1 \\
\text { considering } \\
\text { transaction cost }\end{array}$ & $1.0 \mathrm{E}-4$ & $5.5 \mathrm{E}+0$ & $7.9 \mathrm{E}-1$ & $7.5 \mathrm{E}-5$ & $5.5 \mathrm{E}+0$ & 7.3E-1 & $7.0 \mathrm{E}-5$ & $6.2 \mathrm{E}+0$ & $5.5 \mathrm{E}-1$ & $3.0 \mathrm{E}-4$ & $1.6 \mathrm{E}+1$ & $2.6 \mathrm{E}+0$ & $1.7 \mathrm{E}-4$ & $1.5 \mathrm{E}+1$ & $3.2 \mathrm{E}+0$ \\
\hline $\begin{array}{l}\text { HS-TLBO for } \\
\text { constraint } 2 \\
\text { considering } \\
\text { transaction cost }\end{array}$ & $1.1 \mathrm{E}-4$ & $\underline{2.6 \mathrm{E}+0}$ & $9.6 \mathrm{E}-1$ & $2.0 \mathrm{E}-4$ & $1.1 \mathrm{E}+1$ & $1.6 \mathrm{E}+0$ & $9.3 \mathrm{E}-5$ & $7.4 \mathrm{E}+0$ & 7.1E-1 & $2.0 \mathrm{E}-4$ & $1.3 \mathrm{E}+1$ & $1.9 \mathrm{E}+0$ & $1.6 \mathrm{E}-4$ & $1.6 \mathrm{E}+1$ & $7.3 \mathrm{E}+0$ \\
\hline
\end{tabular}


Solving Complex Cardinality Constrained Mean-variance Portfolio Optimization Problems Using Hybrid HS and TLBO Algorithm
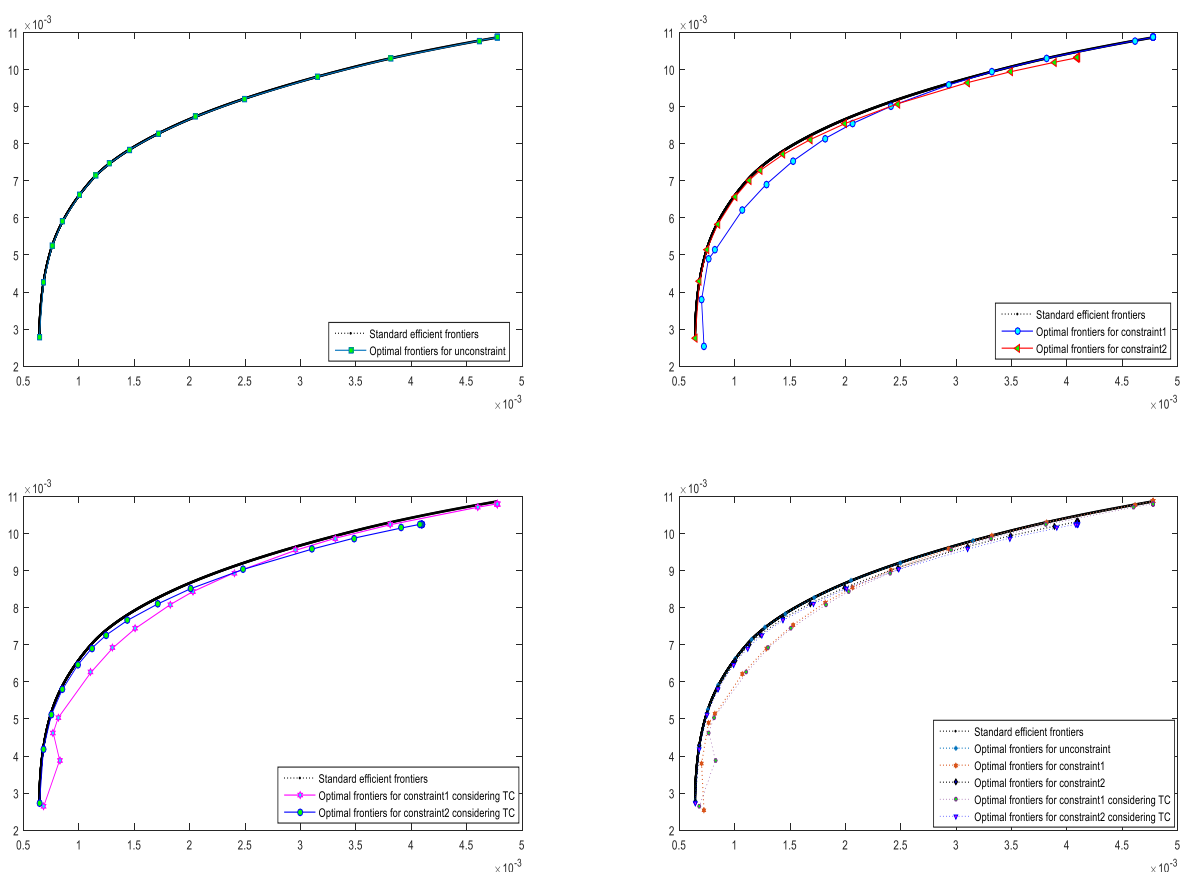

Figure 5. Comparison of efficient frontier on HangSengtest data
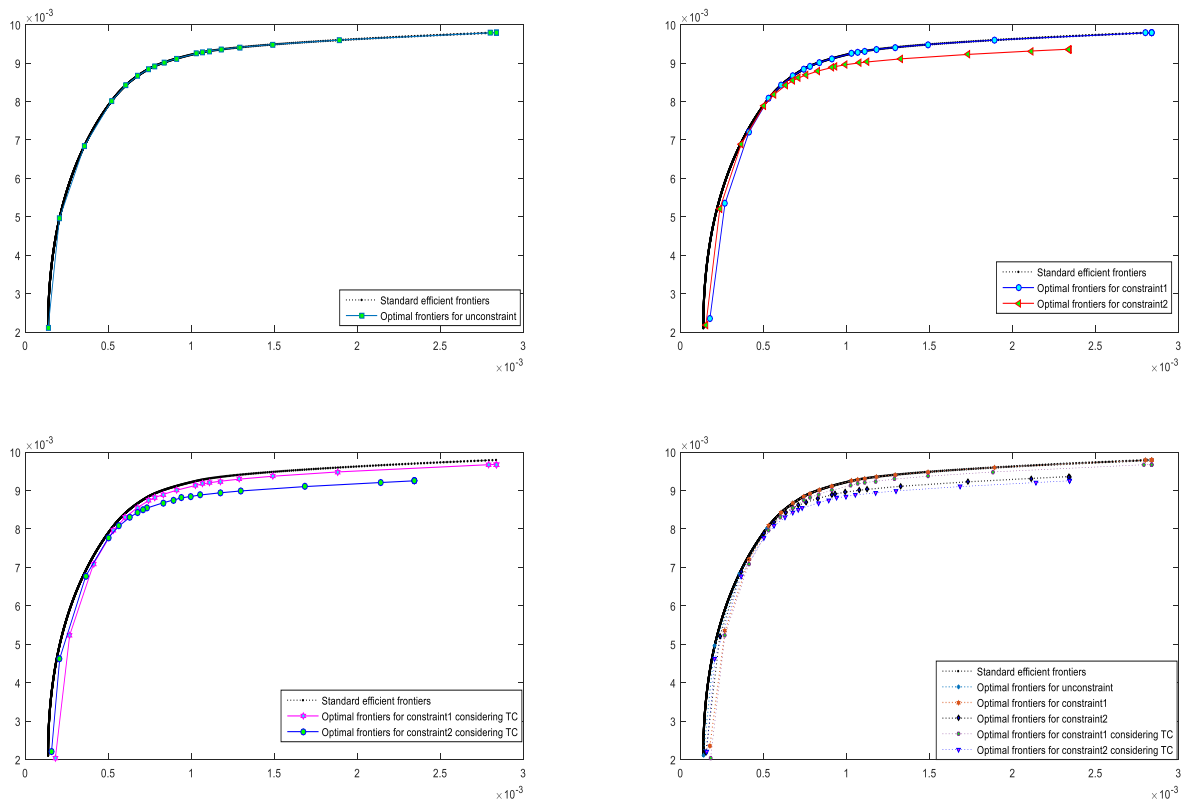

Figure 6. Comparison of efficient frontier on DAX100 test data

DOI: $10.24818 / 18423264 / 52.3 .18 .16$ 
Shouheng Tuo, Hong He
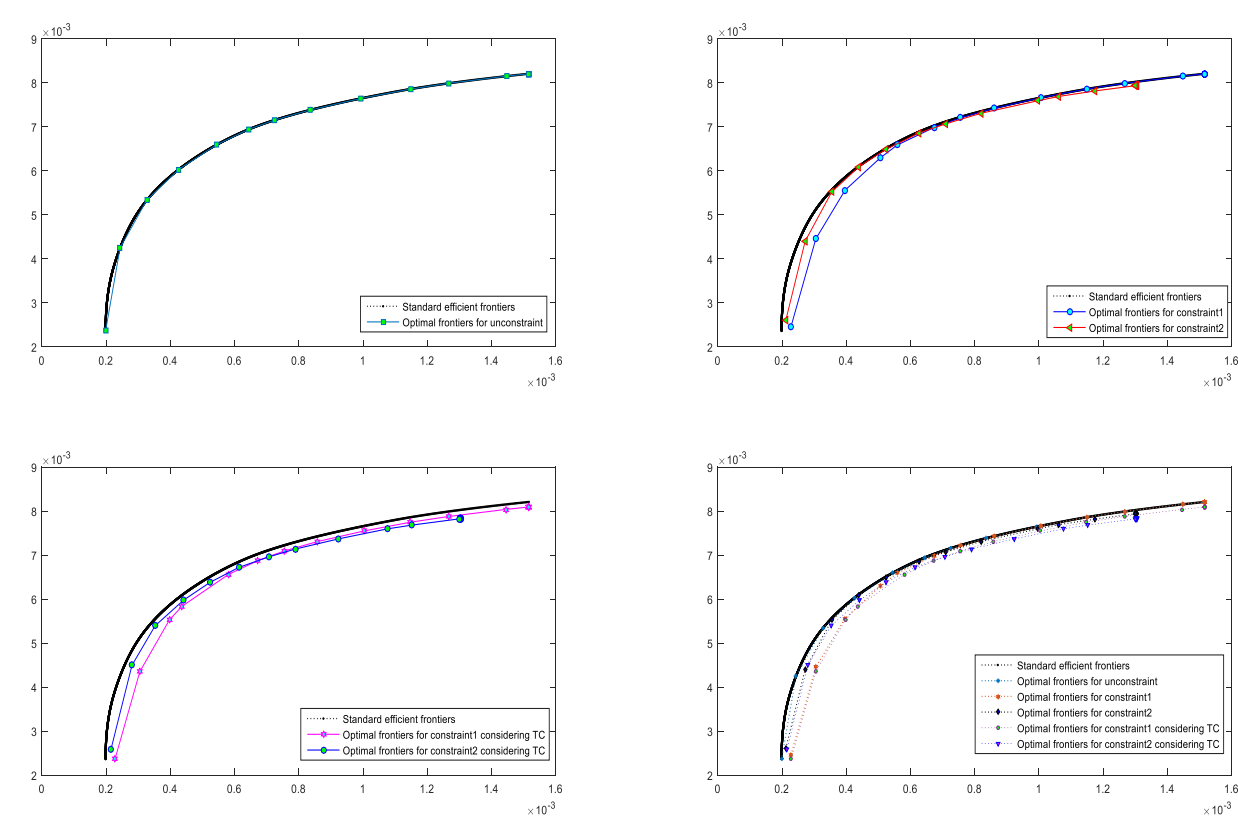

Figure 7. Comparison of efficient frontier on FTSE100 test data
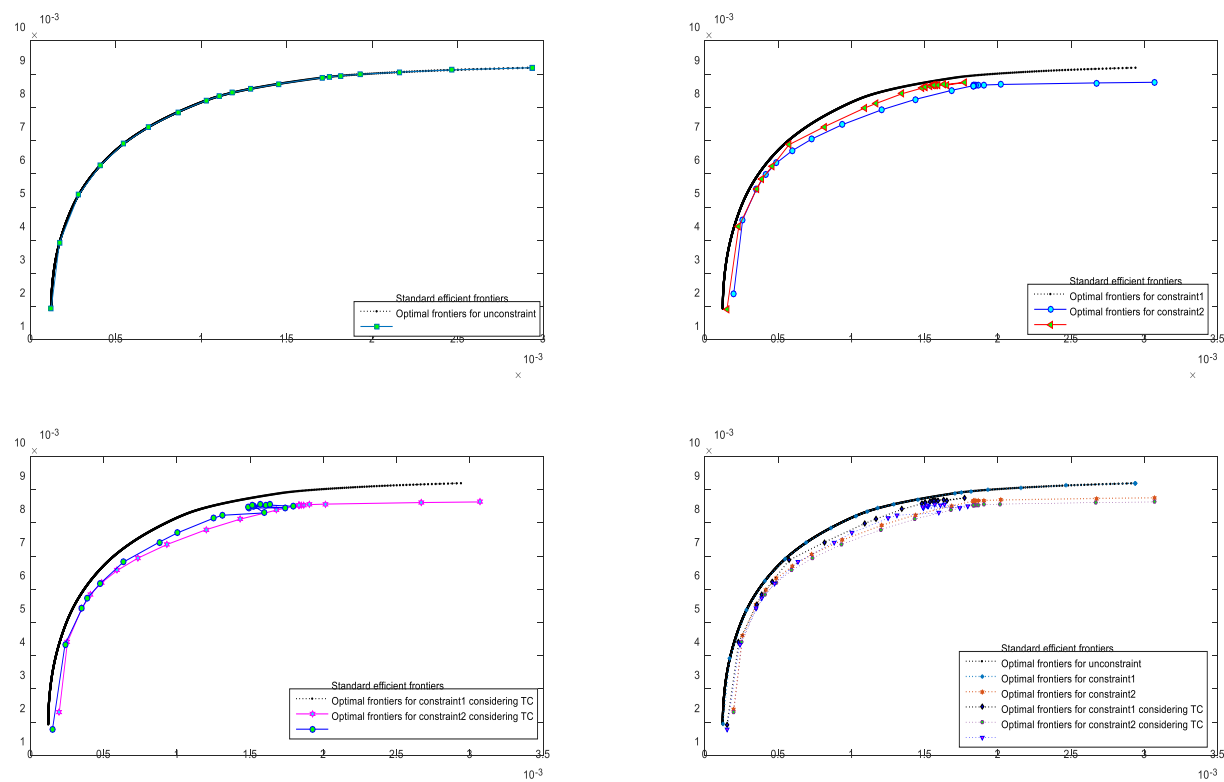

Figure 8. Comparison of efficient frontier on S\&P100 test data 
Solving Complex Cardinality Constrained Mean-variance Portfolio Optimization Problems Using Hybrid HS and TLBO Algorithm
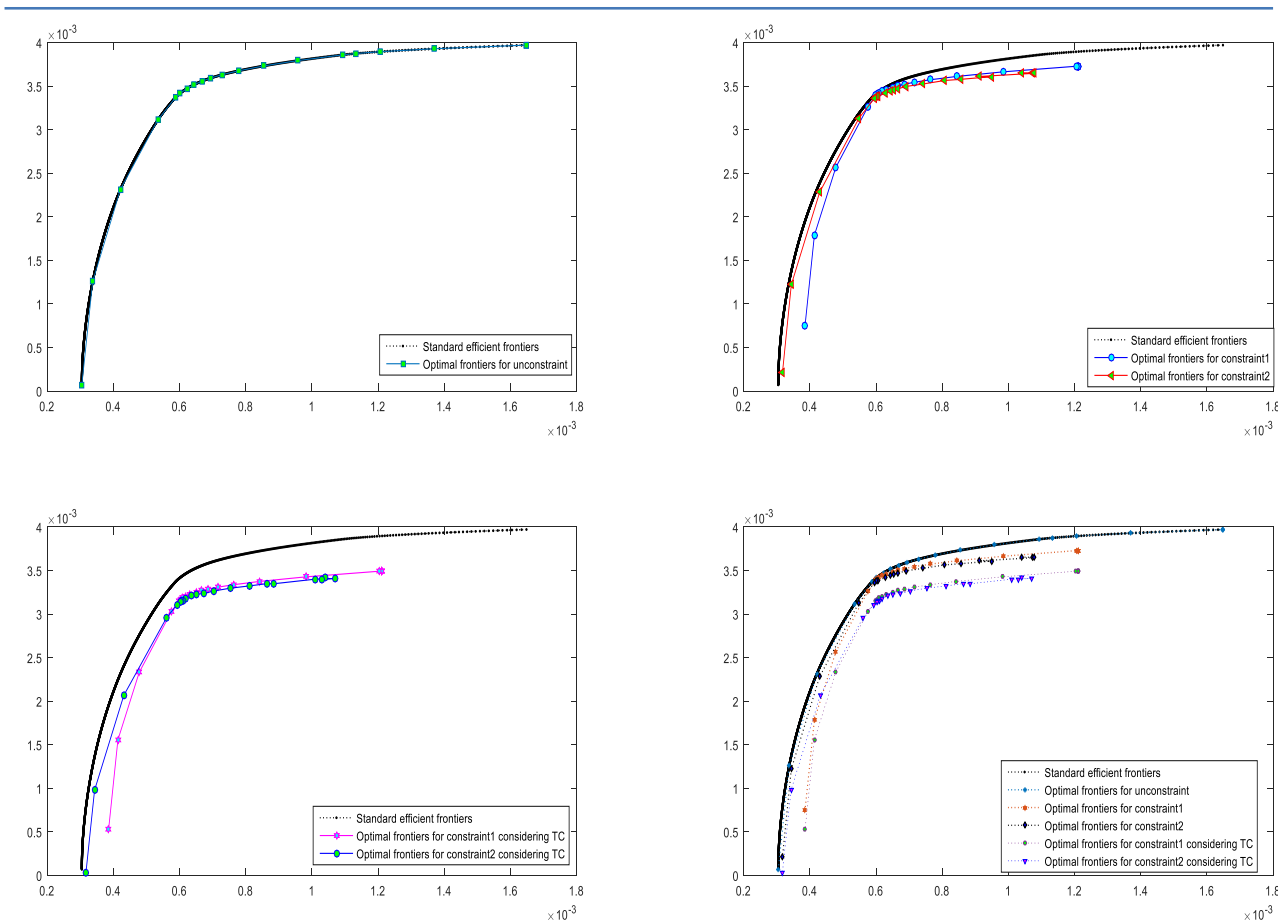

Figure 9. Comparison of efficient frontier on Nikkei225 test data

From Table1, it can be seen obviously that, for five datasets, our method on all the metrics (MED, VRE, MRE) are superior to other four algorithms.

In Table 2, our method also has evident advantages on MED over other algorithms, and the HS-TLBO is superior to comparison methods for most of data sets.

As shown in Fig.5-Fig.9, for unconstrained CCMV model, the efficient frontier of our method is overlapped with the standard efficient frontier almost on all five datasets, which demonstrates that our method is effective for solving unconstrained CCMV models. For constraint1, constraint2, contraint 1 considering TC and constraint 2 considering TC, the proposed HS-TLBO algorithm shows very high performance, the corresponding optimal frontiers are very close to the standard efficient frontiers obtained without considering any constraints.

\section{CONCLUSION}

DOI: $10.24818 / 18423264 / 52.3 .18 .16$ 
Shouheng Tuo, Hong He

In this work, we focus on solving large scale complex portfolio optimization problems which consider the constraint of portfolio selection proportion of each asset and the transaction costs. Firstly, we introduce the mathematical model of portfolio selection and the CCMV model which considers the constraint of transaction cost. Related works for solving the portfolio selection problems are analysed. Secondly, we highlight the proposed HS-TLBO algorithm for solving the complex portfolio optimization problems. Finally, five experiments are performed to investigate the performance of HS-TLBO. The experimental results demonstrate that our method has the obvious advantage on solving complex portfolio selection problems over four state-of-the-art intelligent algorithms.

\section{ACKNOWLEDGEMENTS}

The author would like to thank all the editors, reviewers and referees for their valuable comments. This work was supported by the National Natural Science Foundation of China under Grant Nos.11401357, Social Science Foundation of The Chinese Education Commission (17YJC790048) and Social Science Foundation of Shaanxi Province, China (2015D030).

\section{REFERENCE}

[1] Markowitz, H. M. (1959), Portfolio Selection: Efficient Diversification of Investments. John Wiley \& Sons;

[2] Bertsimas, D. and Shioda, R. (2009), Algorithm for Cardinality-constrained Quadratic Optimization. Computational Optimization and Applications, 43(1):1-22;

[3] Lobo, M. S., Fazel, M. and Boyd, S. (2007), Portfolio Optimization with Linear and Fixed Transaction Costs. Annals of Operation Research, 152(1):341-365;

[4] Patel, N. R. and Subrahmanyam, M. G. (1982), A Simple Algorithm for Optimal Portfolio Selection with Fixed Transaction Costs. Management Science, 28(3):303-314;

[5] Perold, A. F. (1984), Large-scale Portfolio Optimization. Management Science, 30(10):1143-1160;

[6] Shaw, D. X., Liu, S. and Kopman, L. (2008), Lagrangian Relaxation Procedure for Cardinality-constrained Portfolio Optimization. Optimization Methods and Software, 23(3):411-420;

[7] Gaspero, L. D., Tollo, G. D., Roli, A. and Schaerf, A. (2010), Hybrid Metaheuristics for Constrained Portfolio Selection Problems. Quantitative Finance, 11(10):1-15; 
Solving Complex Cardinality Constrained Mean-variance Portfolio Optimization Problems Using Hybrid HS and TLBO Algorithm

[8] Borchers, B. and Mitchell, J. E. (1994), An Improved Branch and Bound Algorithm for Mixed Integer Nonlinear Programs. Computers and Operations Research, 21(4):359-367;

[9] Konno, H. (1990),Piecewise Linear Risk Function and Portfolio Optimization. Journal of the Operations Research Society of Japan, 33(2):139-156;

[10] Konno, H. and Yamazaki, H. (1991), Mean-absolute Deviation Portfolio Optimization Model and its Applications to Tokyo Stock Market. Management Science, 37(5):519-531;

[11] Z.W. Geem, J. Kim, G. Loganathan (2001), Music-inspired Optimization Algorithm Harmony Search; Simulation, 76:60-68;

[12] Rao R. V., Savsani V. J, Vakharia D. P. (2012), Teaching-Learning-Based Optimization: An Optimization Method for Continuous Non-linear Large Scale Problems[J]. Information Sciences, 183(1):1-15;

[13]Bertsimas, D. and Shioda, R. (2009), Algorithm for Cardinality-constrained Quadratic Optimization. Computational Optimization and Applications, 43(1):1-22;

[14] Bienstock, D. (1995), Computational Study of a Family of Mixed-integer Quadratic Programming Problems. Mathematical programming, 74(2):121140 ;

[15] Chang, T.-J., Meade, N., Beasley, J. E. and Sharaiha, Y. M. (2000),Heuristics for Cardinality Constrained Portfolio Optimization. Computers and Operations Research, 27(13):1271-1302;

[16] Soleimani, H., Golmakani, H. R. and Salimi, M. H. (2009), Markowitz-based Portfolio Selection with Minimum Transaction Lots, Cardinality Constraints and Regarding Sector Capitalization Using Genetic Algorithm. Expert Systems and Applications, 36(3):5058-5063;

[17] T.Cura(2009),Particle Swarm Optimization Approach to Portfolio Optimization; Nonlinear Anal.:Real.WorldAppl.10(4):2396-2406;

[18] G.-F.Deng,W.-T.Lin,C.-C.Lo(2012), Markowitz-based Portfolio Selection with Cardinality Constraints Using Improved Particle Swarm Optimization; Expert.Syst.Appl. 39(4)4558-4566;

[19] Jing Chen;Quan-kePan;Jun-qing Li (2012), Harmony Search Algorithm with Dynamic Control Parameters; Applied Mathematics and Computation,No.2,592-604;

[20] Pouya A. R., Solimanpur M., Rezaee M. J. (2016), Solving Multi-objective Portfolio Optimization Problem Using Invasive Weed Optimization [J]. Swarm \& Evolutionary Computation, 28:42-57;

[21] Babaei S., Sepehri M. M., Babaei E. (2015), Multi-objective Portfolio Optimization Considering the Dependence Structure of Asset Returns [J]. European Journal of Operational Research, 244(2):525-539;

[22] Zhen Wang (2012), Models and Algorithms for Some Kinds of Portfolio Optimization Problems[D], Xidian University, 2012 (Chinese);

DOI: 10.24818/18423264/52.3.18.16 
Shouheng Tuo, Hong He

[23] Tuo S. H. (2016), A Modified Harmony Search Algorithm for Portfolio Optimization Problems[J]. Economic Computation \& Economic Cybernetics Studies \& Research, 50(1); ASE Publishing, Bucharest. 